\title{
Electrical Characteristics and Electrohydrodynamic Flows in Electrostatic Precipitator of Six Shaped Discharge Electrodes
}

\author{
H. Shen, H. Jia and Y. Kang ${ }^{\dagger}$ \\ School of Environmental Science and Engineering, Donghua University, Shanghai, 201620, China \\ †Corresponding Author Email: ymkang@dhu.edu.cn
}

(Received October 22, 2019; accepted May 7, 2020)

\begin{abstract}
The electrical characteristics and electrohydrodynamic (EHD) flows in a wire-plate electrostatic precipitator with six shaped discharge electrodes are analyzed by employing the commercial software ANSYS FLUENT with the aid of User Defined Function (UDF). The results show that the corona position of the discharge electrode plays important roles in generating space charge distribution. When the inlet velocity is relatively low, the vortexes induced by the secondary flow exist not only in the downstream of the discharge electrode, but also in the vicinity of the collecting plate. The vortex near the collecting surface in the Knife-shaped system produces the highest recirculation velocity and turbulence intensity, and covers the widest region among the six configurations. For the high inlet velocity, the local recirculation and high turbulence by the secondary flow near the collecting plate in the all six channels disappear but still remain in the downstream of the discharge electrodes, and the airflow in the central region of the six channels would be accelerated. The highest vortex strength and turbulence intensity and the strongest speed-up effect occur in the center of the channel with the Knife-shaped discharge electrode compared with the cases of the other five systems.
\end{abstract}

Keywords: Electrostatic precipitator; Electrohydrodynamics (EHD); Vortex structure; Secondary flow; Discharge electrode.

\section{NOMENCLATURE}

De $\quad$ ion diffusivity

$F_{i} \quad$ convective flux in the ith direction

$E_{i} \quad$ electric field strength in the $i$ th direction

$H \quad$ height of corona electrode

I specific current of discharge wire

$J \quad$ average current density on the collecting electrode

turbulent kinetic energy

ion mobility

pressure

radius of discharge wire

radius of Star electrode tip

concave surface radius of Star electrode

source term in standard scalar transport

equation

distance of wire-to-wire

distance of wire-to-plate

gas velocity at the inlet

gas velocity component in the $k$ th direction

$U_{0} \quad$ applied electric potential at the discharge electrode

$W \quad$ wide of corona electrode

$x, y, z \quad$ cartesian coordinate

$\Gamma_{\phi}$

$\varepsilon$

$\varepsilon_{0}$

$\mu$

$\mu_{t}$

$\mu_{t}$

pio

pion

$\rho_{w}$

$\varphi$

$\phi \quad$ scalar

\section{diffusion coefficient}

dissipation rate of $k$

permittivity of free space

kinematic viscosity

turbulent eddy viscosity

gas density

ion charge density

space charge density of discharge electrode surface

electric potential 


\section{INTRODUCTION}

Environmental protection, especially air pollution control, has become a crucial problem of public concern in China, because the energy consumption as well as the emission of environmental pollutants has grown dramatically with the rapid economic development (Fu et al. 2013; Xue et al. 2016; Sun et al. 2018). Electrostatic precipitation constitutes the main method for removing hazardous particles from exhaust gases originating from various production installations such as power plants, mining installations or chemical industries (Parker 1997), and numerous investigations have shown that the electrode configuration and arrangement play important roles for improving particle collection efficiency in an electrostatic precipitator (ESP) (Lagarias 1960; Jarzebski and Chojnowski 1974; Kim et al. 1998; Jędrusik et al. 2001; Brocilo et al. 2001; Chun et al. 2007; Lei et al. 2008; Neimarlija et al. 2011; Sander et al. 2018). As an initial approach, some experimental studies have been conducted on the voltage-current (i.e., V-I) characteristics and ESP efficiency with different shaped electrodes (Lagarias 1960; Jarzebski and Chojnowski 1974). Unfortunately, it is difficult to measure the electrical and flow fields inside the ESPs directly because of the presence of corona discharge (Lei et al. 2008). Therefore, numerical simulation has been widely used for analyzing the electrical characteristics and airflow behaviors in the ESPs. For instance, Brocilo et al. (2001) developed a numerical model to examine the effects of discharge and collecting electrode geometries on the electric field and ion density distributions in an ESP with three type (so-called I-type, U-type and C-type) of collecting electrodes, as well as the effect of corona wire configuration on collection efficiency of submicron particles. Neimarlija et al. (2011) used a finite volume discretization of the solution domain by arbitrary polyhedral control volumes as a numerical method for calculating the electrical characteristics in the spike-plate electrode precipitator.

However, particle collection in the ESP channel is an extremely complicated process which is affected by the electrostatic field together with the electrohydrodynamic (EHD) flow and many other factors. As the phenomena occur in other processes with the EHD effects in channels (Sugiyama et al. 2013; Rouhollahi et al. 2018), flow interaction between the mainstream (i.e., primary flow) and the secondary flow (i.e., electric wind or ionic wind induced flow) in the wire-plate ESP channel (with smooth corona wire) has been investigated numerically and experimentally for many years, such as the studies by Yamamoto et al. (1981) and Killio et al. (1992), and more details on this subject in the past decades have been reviewed by Adamiak (2013). In these investigations, most of the numerical studies considered the simplified case of traditional smooth wire electrode with homogeneous discharge, only in a few studies more complicated shape of discharge electrodes were investigated, such as the spiked-type corona electrode. Brocilo et al. (2004) investigated the EHD flow patterns in a wide spacing spike-plate electrostatic precipitator with negative corona, and reported that the ion flow patterns may induce transverse recirculation vortices associated with each discharge point. Farnoosh et al. (2011) developed a three-dimensional numerical model and performed a detailed investigation on the electrical and the EHD characteristics of a single spiked wireplate electrostatic precipitator. Podlinski et al. (2013) showed that when a spiked electrode is used, negative corona discharge is more efficient than positive corona discharge for collecting submicron particles by generating a higher amount of ionic charges to the channel.

Although previous studies have gained better understandings of the coupling flow in the wireplate (smooth wire) ESP, less information about other discharge electrodes, which are used in many industrial ESPs, is available. Therefore, the wireplate ESP channels with six shapes of discharge electrodes (i.e., round-, trap-, square-, knife-shaped and two star-shaped) is investigated by numerical simulation. The User Defined Function (UDF) of FLUENT code combined with turbulence model is adopted to address the electrical characteristics and the EHD flow in the ESP channel. The distributions of electrical characteristics, gas velocity and turbulence intensity in the wire-plate ESP channels of the six shaped discharge electrodes with typical electrical and airflow conditions are also analyzed.

\section{Configuration OF Discharge ELECTRODE}

The ESP channel consists of two collecting plates through which the dust-laden gas is passing, and a series of discharge electrodes are placed in the middle plane between the two plates. The electrical characteristics and the gas flow patterns in the ESP are simulated by the present numerical model for six shaped discharge electrodes commonly used in ESPs (see Fig. 1). The geometric structures of the discharge electrodes are shown in Fig. 1(a), with (i) to (vi) represent the six kinds of discharge electrode.

Similar to some previous works of modeling the EHD flow in wire-plate ESPs (Yamamoto and Velkoff 1981; Kallio and Stock 1992; Neimarlija et al. 2011; Adamiak 2013), the ESP channel is simplified to be a three-corona-wire configuration, see Fig. 1(b). In order to eliminate the influence of the inlet and outlet boundary conditions on the numerical results, the distances between both the first corona wire and the inlet and the third wire and the outlet of the calculation domain are both set to be $0.12 \mathrm{~m}$ (i.e., $S_{x}$, the wire-to-wire spacing), and flat-plate is considered as the collecting electrode. The EHD flow in the ESP channel is assumed to be fully developed incompressible, isothermal and isotropic turbulent flow. Moreover, due to the symmetry of the channel's geometrical configuration, the computational domain is reduced to only the upper half of the channel between the 


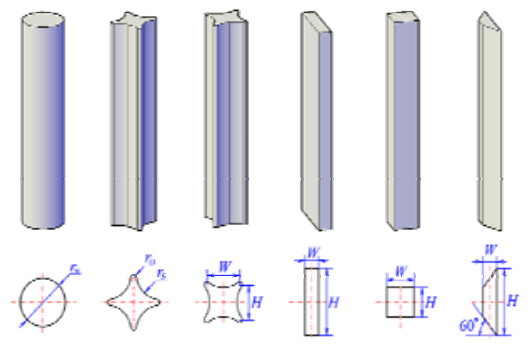

(i) Round (ii) Star-1 (iii) Star-2 (iv) Trap (v) Square (vi) Krife

a) Six-shaped discharge electrodes

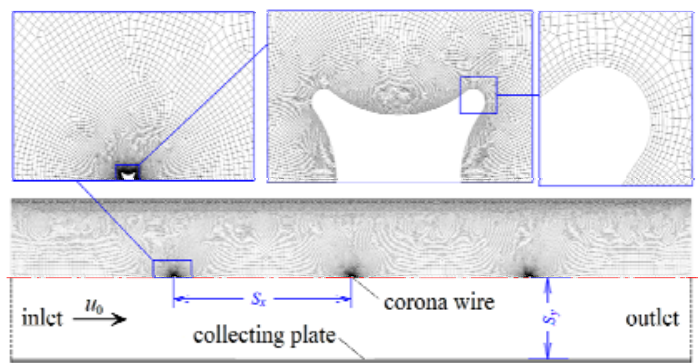

b) Numerical domain with refined mesh near the wire of Star-2 system

Fig. 1. Configurations of discharge electrode and computational domain of the ESP channel.

Table 1 Main parameters of the ESP channel for simulation.

\begin{tabular}{|c|c|c|c|c|c|c|c|}
\hline Discharge electrode & $r_{\mathrm{W}} / \mathrm{mm}$ & $r_{\mathrm{D}} / \mathrm{mm}$ & $r_{\mathrm{S}} / \mathrm{mm}$ & $\mathrm{W} / \mathrm{mm}$ & $\mathrm{H} / \mathrm{mm}$ & $2 S_{x} / \mathrm{mm}$ & $2 S_{y} / \mathrm{mm}$ \\
\hline Round & 2.0 & - & - & - & - & 240 & 200 \\
\hline Star-1 & - & 0.3 & 8 & 4 & 4 & 240 & 200 \\
\hline Star-2 & - & 0.3 & 8 & 4 & 4 & 240 & 200 \\
\hline Trap & - & - & - & 1.5 & 7 & 240 & 200 \\
\hline Square & - & - & - & 3 & 3 & 240 & 200 \\
\hline Knife & - & - & - & 1.5 & 7 & 240 & 200 \\
\hline
\end{tabular}

collecting plate and discharge electrodes. As an example of the all cases, the computational domain of the channel with three Star-shaped corona wires is shown in Fig. 1(b).

The main parameters used for numerical simulations are summarized in Table 1 (Lagarias 1960; Jędrusik et al. 2001; Brocilo et al. 2001), where $S_{\mathrm{x}}$ and $S_{\mathrm{y}}$ are the wire-to-wire and wire-toplate distances, respectively. $r_{\mathrm{w}}$ is the radius of the smooth corona wire (Fig. 1(a)-(i)), $r_{\mathrm{D}}$ is the radius of the Star electrode tip, and $r_{\mathrm{s}}$ is the concave surface radius of the Star electrode (Figs. 1(a)-(ii) and 1(a)-(iii)). $W$ and $H$ are the width and height of the corona electrodes (Figs. 1(a)-(iii) to 1(a)-(vi)), respectively.

\section{DESCRIPTION OF THE NUMERICAL MODEL}

\subsection{Governing Equations}

The electrical characteristics in an ESP channel can be determined by Poisson's and current-continuity equations, and the EHD turbulent flow in the channel is governed by the time-averaged mass continuity and Navier-Stokes equations including a turbulent model and the influence of electrically induced body force. Thus the governing equations are as follows (Yamamoto and Velkoff 1981; Kallio and Stock 1992; Brocilo et al. 2004; Farnoosh et al. 2011; Tathiri et al. 2017; Rouhollahi et al. 2018):

Poisson's equation

$$
\nabla^{2} \varphi=-\rho_{\text {ion }} / \varepsilon_{0}
$$

Current continuity equation $\frac{\partial}{\partial x_{k}}\left(\rho \rho_{\text {ion }}\left(k_{\text {ion }} E_{k}+u_{k}\right)\right)=\rho D_{\mathrm{e}} \frac{\partial^{2} \rho_{\text {ion }}}{\partial x_{k}^{2}}$

Conservation of mass

$\frac{\partial}{\partial x_{k}}\left(\rho u_{k}\right)=0$

Conservation of momentum

$\frac{\partial}{\partial x_{k}}\left(\rho u_{i} u_{k}-\left(\mu+\mu_{\mathrm{t}}\right) \frac{\partial u_{i}}{\partial x_{k}}\right)=-\frac{\partial p}{\partial x_{k}}+\rho_{\text {ion }} E_{i}$

where $\varphi$ is the electric potential, $\rho_{\text {ion }}$ is the ion charge density, $\varepsilon_{0}$ is the permittivity of free space, $\rho$ is the gas mass density, $k_{\text {ion }}$ is the ion mobility, $u_{k}$ is the component of gas velocity in the $x_{k}$ direction, $D_{\mathrm{e}}$ is the ion diffusivity, $\mu$ and $\mu_{\mathrm{t}}$ are the gas dynamic viscosity and turbulent dynamic viscosity, respectively. $p$ is the gas pressure, $E_{k}$ is the component of the electric field strength in the $x_{k}$ direction. The coupling between the electric field and the turbulence flow is the electrical body force, i.e., $\rho_{\mathrm{ion}} E_{i}$, and it is introduced into the momentum equation (Eq. (4)) via a User Defined Function. The momentum coupling has been found to be significant when the secondary flow produced by ionic wind is considerably larger compared to the inlet gas velocity (Yamamoto and Velkoff 1981; Kallio and Stock 1992; Leonard et al. 1983)

Many turbulence models including the Standard $k-\varepsilon$ model (Feng et al. 2018) and the RNG $k-\varepsilon$ model (Choi and Fletcher 1998; Park and Kim 2000) have been widely used in previous studies to solve the EHD turbulence flow. Although Feng et al. (2018) simulated the EHD flow in the ESP channel with cavity walls by employing six turbulence models, 
and found that both the RSM model and the Standard $k-\varepsilon$ model are appropriate for simulating the time-averaged velocity, Choi and Fletcher (1998) showed that for the computational cost and numerical accuracy, the RNG $k-\varepsilon$ model is an reasonable choice in the EHD modeling of the ESP. Park and Kim (2000) also compared the simulation results of the RNG $k-\varepsilon$ model with experimental data, and the results proved that the RNG $k-\varepsilon$ model was suitable for simulating the EHD turbulent flow. In addition, as the Reynolds number in a real ESP channel is between $5 \times 10^{3}$ and $2.5 \times 10^{4}$, the lowReynolds number effects need to be considered in modeling the turbulent flow. Comparing with the Standard $k-\varepsilon$ model, the RNG $k-\varepsilon$ model gives remarkable improvements in the treatment of massive flow separation and anisotropic turbulence (Park and Kim 2000). Therefore, the RNG $k-\varepsilon$ model is adopted in the present study.

To predict the turbulent flow in the ESP channel with the ionic wind, the commercial FLUENT code (ANSYS FLUENT v19.3.0) is employed to complete the solution. However, Eqs. (1) and (2), and the term of the electrical body force, i.e., $\rho_{\mathrm{ron}} E_{i}$ in Eq. (4), are not contained in the model of the FLUENT. For this reason the electric field intensity and the distribution of ion charge density are firstly determined from the numerical solution of the Poisson's equation and the current continuity equation by employing the finite volume method. Therefore, a UDF is developed and used for linking the solution of the electric field with the FLUENT code, and Eqs. (1) and (2) are transformed into the standard scalar transport equation format of FLUENT code, which can be written as:

$$
\frac{\partial(\rho \phi)}{\partial t}+\nabla \cdot\left(F_{i} \phi\right)=\nabla \cdot\left(\Gamma_{\phi} \nabla \phi\right)+S_{\phi}
$$

Terms of the scalar transport equation are listed in Table 2, where $\phi$ is the scalar of interest, $F_{i}$ is the convective flux, $\Gamma_{\phi}$ is diffusion coefficient of $\phi$, and $S_{\phi}$ is the source of $\phi$.

Table 2 Terms of the scalar transport equation

\begin{tabular}{|c|c|c|c|c|}
\hline Equation & $\phi$ & $F_{i}$ & $\Gamma_{\phi}$ & $S_{\phi}$ \\
\hline$(1)$ & $\varphi$ & 0 & 1 & $\rho_{\text {ion }} / \varepsilon 0$ \\
\hline$(2)$ & $\rho_{\text {ion }}$ & $k_{\text {ion }} E_{k}+u_{k}$ & $D_{\mathrm{e}}$ & 0 \\
\hline
\end{tabular}

\subsection{Boundary Conditions and Grid Independency Test}

Boundary conditions used in the simulations are summarized in Table 3 . The inlet and outlet of the channel are set as Velocity-inlet and Pressureoutlet, respectively. The discharge electrode and the collecting electrode are modelled as Wall boundaries, and other surfaces are treated as symmetry planes.
Table 3 Summary of boundary conditions

\begin{tabular}{|c|c|c|c|}
\hline Equation & $\begin{array}{c}\text { Gas } \\
\text { velocity }\end{array}$ & $\begin{array}{c}\text { Electric } \\
\text { potential }\end{array}$ & $\begin{array}{c}\text { Charge } \\
\text { density }\end{array}$ \\
\hline Inlet & $\begin{array}{c}\text { Velocity } \\
\text { inlet }\end{array}$ & $\partial \varphi / \partial n=0$ & $\partial \rho_{\text {ion }} / \partial n=0$ \\
\hline Outlet & $\begin{array}{c}\text { Pressure } \\
\text { outlet }\end{array}$ & $\partial \varphi / \partial n=0$ & $\partial \rho_{\text {ion }} / \partial n=0$ \\
\hline $\begin{array}{c}\text { Collecting } \\
\text { electrode }\end{array}$ & Wall & $0 \mathrm{kV}$ & $\partial \rho_{\text {ion }} / \partial n=0$ \\
\hline $\begin{array}{c}\text { Discharge } \\
\text { electrode }\end{array}$ & Wall & $50 \mathrm{kV}$ & $\begin{array}{c}\text { Peek } \\
\text { formula }\end{array}$ \\
\hline
\end{tabular}

To achieve converged results of the EHD simulations, the grid independence tests need to be carried out at first to exclude the effect of grid resolution on numerical results. Thus a set of presimulations were performed by computing the same case with different grid numbers in the presimulations, and the quantitative grid convergence was obtained by using the Grid Convergence Index (GCI) proposed by Roache (1994).

The details for the test can be found in our previous study (Shen et al. 2000), and the results with three mesh numbers $\left(N_{1}, N_{2}\right.$ and $\left.N_{3}\right)$ are listed in Table 4. In this study, the relative error $\varepsilon$ rms less than $1 \%$ between the coarse and fine grid is used as the criterion for grid convergence and the equivalent GCI value is $4.35 \%$. Both the GCI and $\varepsilon_{\text {rms }}$ in Table 3 are lower than the criteria values, which suggest that the variables are not influenced by the grid density. Therefore, the final mesh numbers $N$ of the six configurations for all simulations are set at $163,863,164,922,167,577,163,879,158,628$ and 167,828 , respectively.

\subsection{Model Validation}

The experimental model and the test data by AlHamouz et al. (2011) are employed for the validation of the accuracy of the present numerical model, and the details of the experiment can be found elsewhere (Al-Hamouz et al. 2011). The simulated current density on the collecting plate is compared with the experimental data of Al-Hamouz et al. (2011), and the results are shown in Fig. 2, where $J$ is the averaged current density on the collecting electrode.

It is seen from Fig. 2 that the ground plate current density profiles predicted by the present algorithm for two wire-to-wire spacings $\left(S_{x}=0.3 \mathrm{~m}\right.$ and 0.4 $\mathrm{m})$ agree well with the experimental results by AlHamouz et al. (2011).

To validate the turbulence model used for the simulations of the turbulent flow coupled with electric field, Fig. 3 takes the comparison between the numerical simulations of this study and the experimental data by Leonard et al. (1983) under the ionic wind induced turbulent flows with two gas inlet velocities, i.e., $u_{0}=1.0 \mathrm{~m} / \mathrm{s}$ and $2.0 \mathrm{~m} / \mathrm{s}$, respectively. 
H. Shen et al. / JAFM, Vol. 13, No. 6, pp. 1707-1718, 2020.

Table 4 Grid convergence index (GCI) calculations.

\begin{tabular}{|c|c|c|c|c|c|c|c|}
\hline Discharge electrode & $N_{1}$ & $N_{2}$ & $N_{3}$ & $\varepsilon^{21}{ }_{\mathrm{rms}}$ & $\mathrm{GCI}^{21}$ & $\varepsilon^{32} \mathrm{rms}$ & $\mathrm{GCI}^{32}$ \\
\hline Round & 126,560 & 163,863 & 213,689 & $0.25 \%$ & $1.08 \%$ & $0.23 \%$ & $1.00 \%$ \\
\hline Star-1 & 129,078 & 164,922 & 215,398 & $0.35 \%$ & $1.52 \%$ & $0.26 \%$ & $1.13 \%$ \\
\hline Star-2 & 132,850 & 167,577 & 226,850 & $0.82 \%$ & $3.57 \%$ & $1.05 \%$ & $4.56 \%$ \\
\hline Trap & 126,060 & 163,879 & 213,560 & $0.96 \%$ & $4.17 \%$ & $0.75 \%$ & $3.26 \%$ \\
\hline Square & 122,123 & 158,628 & 204,216 & $0.78 \%$ & $3.39 \%$ & $0.63 \%$ & $2.74 \%$ \\
\hline Knife & 133,905 & 167,828 & 228,176 & $1.09 \%$ & $4.74 \%$ & $0.87 \%$ & $3.78 \%$ \\
\hline
\end{tabular}

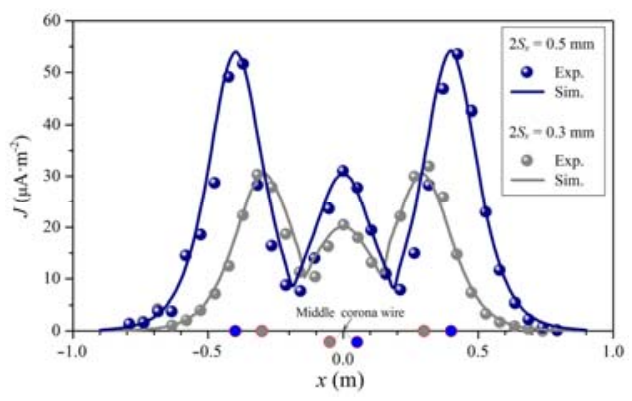

Fig. 2. Comparision of current density distributions on the collecting plate in a wire-plate system between the numerical results and the measured data (Al-Hamouz et al. 2011) $\left(S_{y}=0.16 \mathrm{~m}, r_{\mathrm{w}}=0.85 \mathrm{~mm}\right.$, $\left.U_{0}=-31.5 \mathrm{kV}\right)$.

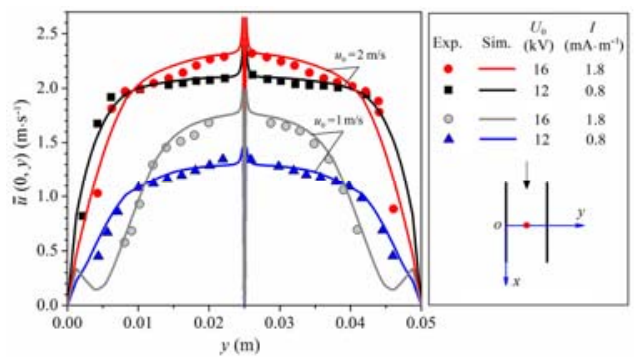

Fig. 3. Comparision of gas velocity profiles in the ESP channel affected by the ionic wind between the numerical results and the experimental data Leonard et al. (1983) $\left(r_{\mathrm{w}}=0.1 \mathrm{~mm}, S_{y}=25 \mathrm{~mm}\right)$.

Figure 3 shows that the velocity distribution at $x=$ 0 along the $y$ direction by the present simulation agrees well with the experimental data of Leonard et al. (1983), where $I$ is the specific current of discharge wire, and the relative error between the experimental and numerical results is $4.23 \%$. It also can be found from Fig. 3 that the increase of gas velocity at the center of channel and the vortexes (local recirculation) induced by the ionic wind near the wall become more evident with the increase of corona current (Yamamoto and Velkoff 1981), meaning that the turbulent model used in the present study can give the accurate and detailed information of EHD flow in the ESP channel.

\section{RESUltS AND DISCUSSION}

\subsection{Electrical Characteristics in the ESP Channels}

The electrical characteristics in the ESP channel for the six shaped discharge electrodes are numerically solved, with the results shown in Fig. 4 to Fig. 8. The geometrical dimensions and electric parameters used for the simulations are presented in Table 1

The electric field intensities $(E)$ in the ESP for the six discharge electrodes show a similar variation tendency in Fig. 4. The electric field intensity around the discharge electrode is higher than that in other regions, and could reach the order of $10^{6}$ $\mathrm{V} / \mathrm{m}$. In the region between two adjacent discharge electrodes, low electric intensity less than $10^{5} \mathrm{~V} / \mathrm{m}$ appears in the channel due to the same polarity of discharge electrodes. The electric strengths near the collecting surfaces facing the discharge electrodes are higher than those of other parts of the collecting plate. For the Knife discharge electrode, the electric field strength near the collecting plate is the highest among the six systems, and the region of strong electric field is also the widest. In contrast, the electric field intensity produced by Round-shaped discharge electrode is the weakest.

Figure 5(a) shows that the electric field strengths at the section $x=0$, for all the six cases, drop dramatically from the maximum values near the discharge electrodes to the minimum values of about $4 \times 10^{5} \mathrm{~V} / \mathrm{m}$ nearly at $y=S_{y} / 4$ and then increase slowly along the $y$ direction. By contrast, it is seen from Fig. 5(b) that, at $x=120 \mathrm{~mm}$, the electric field strengths increase monotonously along the $y$ direction. Comparisons of the electric field strength in the six wire-plate configurations indicate that the electric intensity in the channel with Knifeshaped discharge wire is the highest overall, followed by Trap-shaped electrode, and the electric field strength in the channel with Star-1 wire is the weakest.

Compared to the distributions of the electric field strength, the distribution of space charge density in the channel is affected by the discharge electrode structure more significantly, as shown in Fig. 6. The 


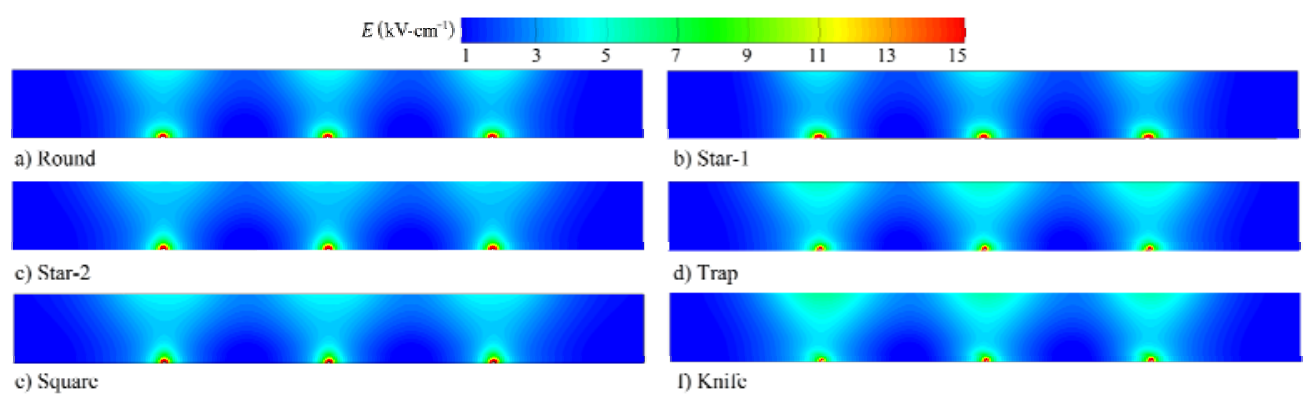

Fig. 4. Electric intensity distributions in the ESP with six-shaped discharge electrodes.

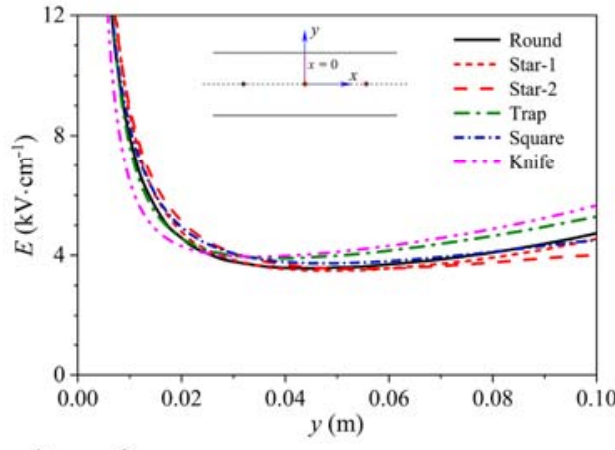

a) $x=0 \mathrm{~mm}$

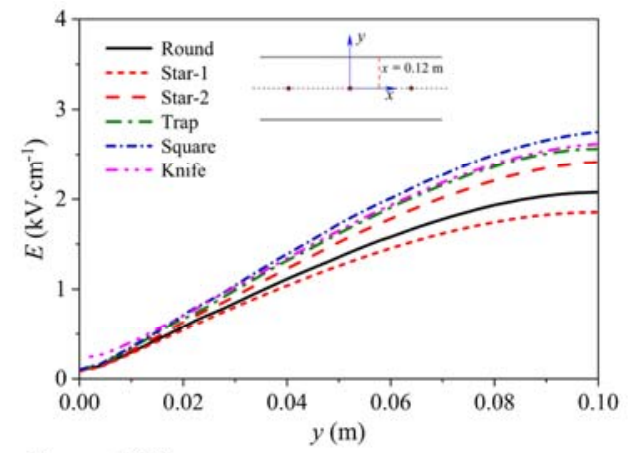

b) $x=120 \mathrm{~mm}$

Fig. 5. Electric intensity distributions of the six discharge electrodes in the ESP channel.

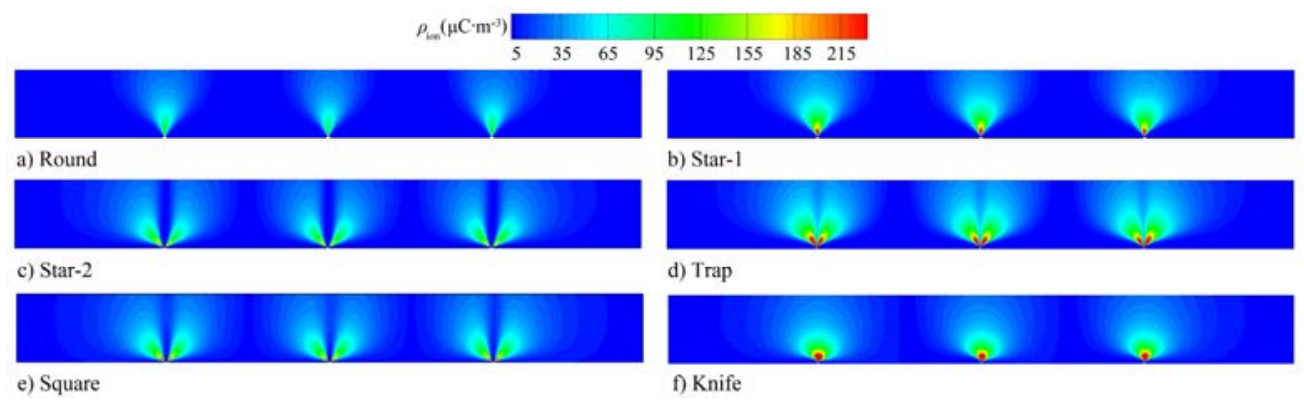

Fig. 6. Current density distributions in the six ESP channels.

charge density gradually increases between the inlet and the first discharging position, and then it forms several high-density regions around the discharging position of electrodes along the $x$ direction, reaching the peak value on the surface of each discharge position. The ionic charge is diffused by the electric field force, so the charge density drops sharply away from the discharge wire, and a low ionic charge density region is formed between two adjacent discharge electrodes.

Figure 7 shows that, in the $y$ direction, the charge density is reduced by two orders of magnitude from the corona wire surface to the collecting plate. The space charge density drops fast in the vicinity of the discharge electrode (Fig. 7(a)), and the closer to the collecting plate, the slower the space charge density decreases. It can be seen from Fig. 7(b) that the charge density between two Star-1 discharge electrodes is the lowest among the six shapes of electrode configurations, followed by Square- and Round-shaped discharge electrodes. Comparison of Figs. 7 (a) and 7 (b) reveals that $\rho_{\text {ion }}$ in the region between two adjacent electrodes is significantly lower than that in other regions. This also illustrates that $\rho_{\text {ion }}$ in the weak region can hardly be increased by changing the discharge electrode structure.

It can be found from Fig. 4 to Fig. 7 that, producing higher electric field strengths and more uniform space charge density distributions than others, Square- and Knife- shaped discharge electrodes are conducive to the driving and trapping of charged particles (White 1963; Parker 1997).

As shown in Fig. 6, the corona position on the electrode surface determines the distribution of 


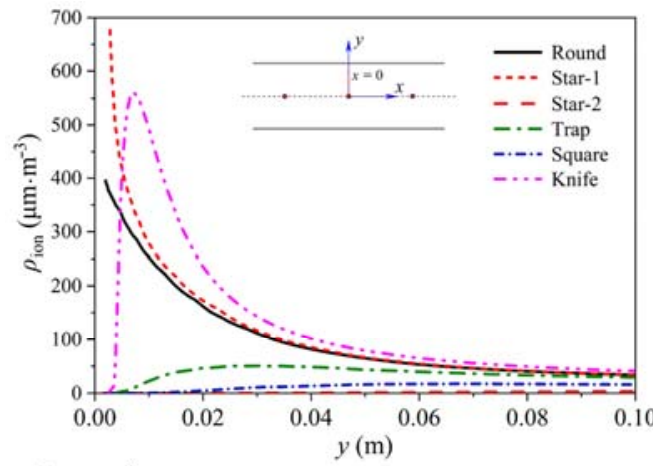

a) $x=0 \mathrm{~mm}$

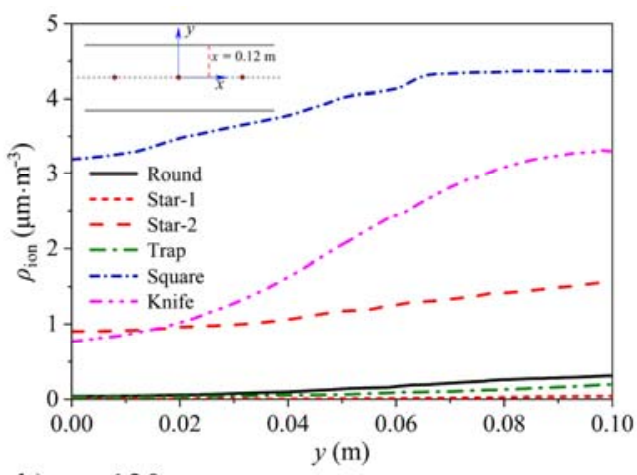

b) $x=120 \mathrm{~mm}$

Fig. 7. Current density distributions on two planes of the six ESP channels.

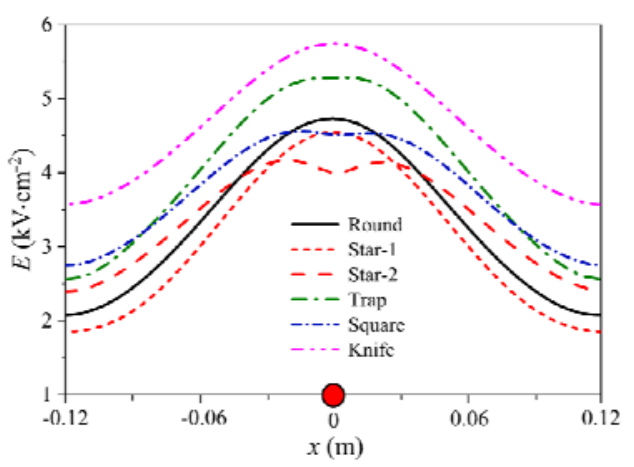

a) Electric field strength

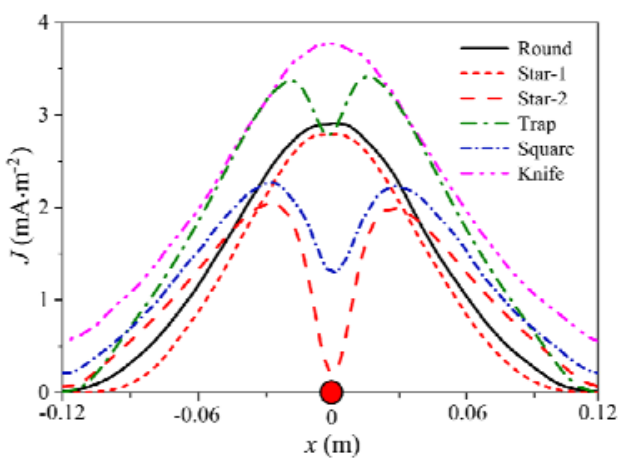

b) Current density

Fig. 8. Electrical characteristics near collecting surface of the six-shaped ESP channels.

space charge density in the channel, so the space charge density distributions in Fig. 7 can be classified into two types. One is produced by the two-point corona, i.e., Star-2, Trap- and Squareshaped discharge electrodes; the remaining is due to the single-point corona. In fact, no corona occurs in the narrow region between the two corona positions of the discharge electrode of the former type (shown in Figs. 6(c), 6(d) and 6(e)), and $\rho_{\text {ion }}$ in the small region is very weak because of the corona shielding effect of two corona positions, which are confirmed by Fig. 7. As seen in Fig. 7(a), the charge density curves of three discharge electrodes with two corona points is close to the horizontal axis at $x$ $=0$, and the curve of Star-2 almost coincides with the horizontal axis due to the largest region without corona. The corona strength of Knife-shaped electrode is the strongest among the electrodes with one-point corona, while Trap-shaped electrode is the strongest in the two-point corona discharge electrodes.

The distributions of electric field intensity and space charge density near the surface of the collecting plate have great influences on the charging and trapping of particles (Parker 1997; Kim et al. 1998). For further comparisons of the electric field and the current density distributions in the six ESP channels, Fig. 8 depicts the profiles of the electric field intensity and current density $(J)$ (at $y=99.75 \mathrm{~mm}$ ) along the collecting surface facing the middle discharge electrode (i.e., the second corona wire).

The results in Fig. 8 show that, for the wires with one corona points, the distributions of $E$ and $J$ along the collecting plate present wave-shaped variations, with peaks of both $E$ and $J$ at the position facing to the wires and the valleys in the midway of two neighboring discharge electrodes. For the wires with two corona points, Fig. 8 shows that there are two peaks for either $E$ or $J$ in such electrode configurations. Moreover, comparing the six curves in Fig. 8(a), it can be found that the electric field strength in Knife-shaped system is the highest, followed by the Trap discharge electrode, and Star1 takes the lowest. The channel with Star-2 discharge electrode has the best uniformity of the electric field distribution due to the smallest peak value, while the worst distribution occurs in Trapshaped configuration. For the current density distributions along the collecting wall in the six channels, see Fig. 8(b), the value of Knife-shaped discharge electrode with single-point corona is the highest, followed by Trap-shaped discharge electrode, and Star-2 takes the lowest. 


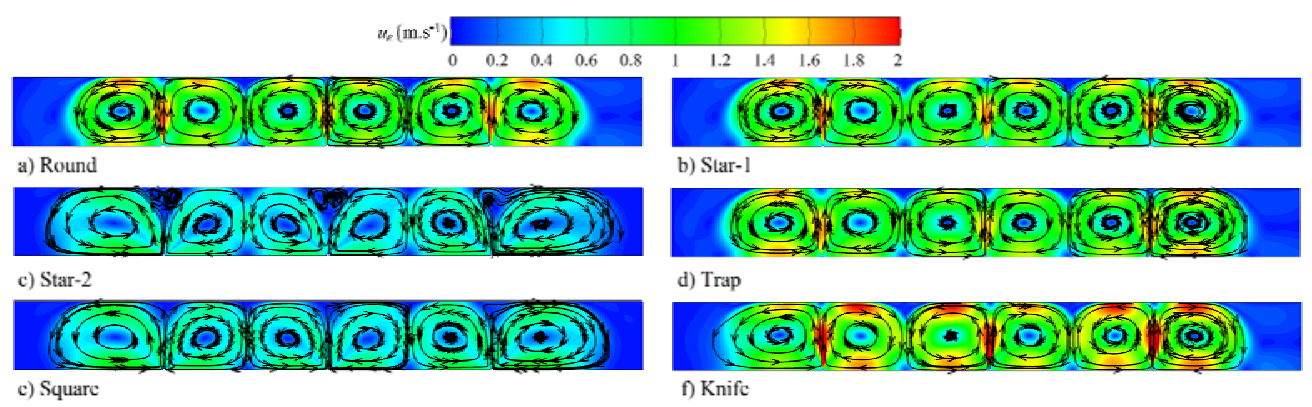

Fig. 9. Comparison of ionic wind induced flows between the six ESP channels at $u_{0}=0 \mathrm{~m} / \mathrm{s}$.

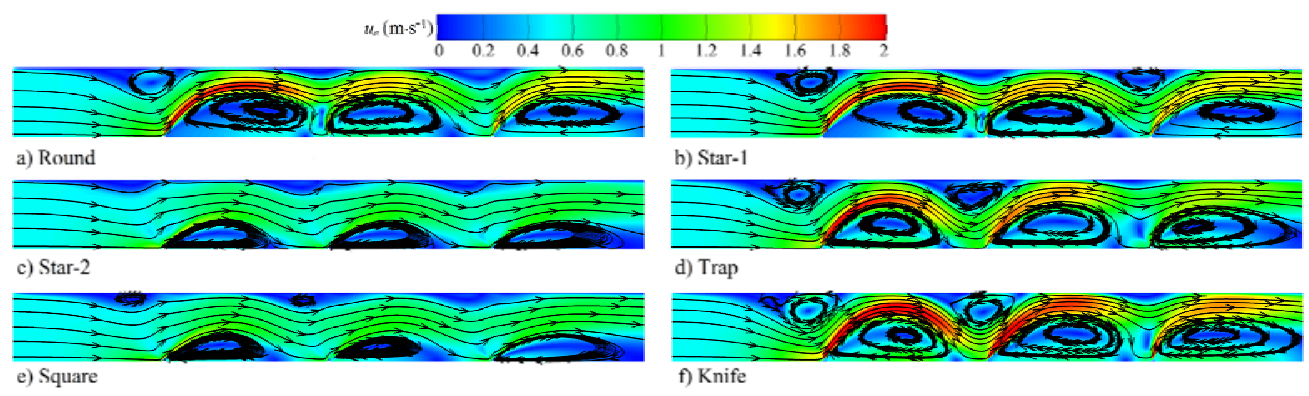

Fig. 10. Comparison of ionic wind induced flows of the six ESP channels at $u_{0}=0.5 \mathrm{~m} / \mathrm{s}$.

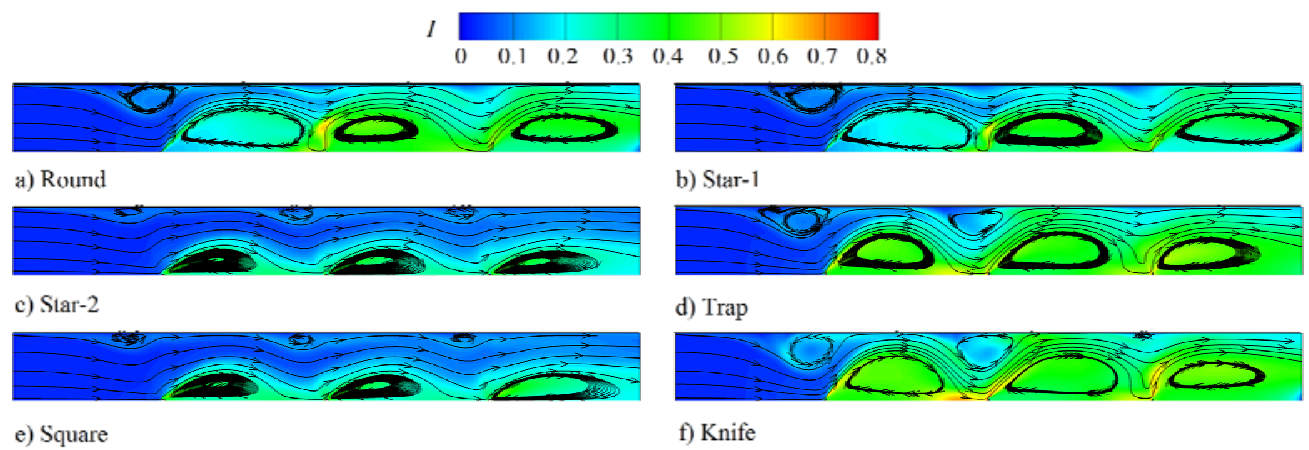

Fig. 11. Turbulence intensities in the six ESP channels at $u_{0}=0.5 \mathrm{~m} / \mathrm{s}$.

\subsection{EHD Flow Induced by Electric Wind}

Particle collection efficiency in an ESP depends not only on the electrical and flow conditions but also on the secondary flow generated by gas ionization, so the characteristics of EHD turbulent flow in an ESP, as well as the ionic wind, plays a crucial role in the collection of charged particles. The EHD flow affects the particle trajectory and capture, and also causes the escape of particles from the collecting plate, particularly in the case of $\mathrm{PM}_{2.5-}$ based small size particles. Therefore, the EHD flows are simulated for four different inlet gas velocities, i.e., $u_{0}=0 \mathrm{~m} / \mathrm{s}, 0.5 \mathrm{~m} / \mathrm{s}, 1.0 \mathrm{~m} / \mathrm{s}$ and $2.0 \mathrm{~m} / \mathrm{s}$. Figures 9 to 15 show the distributions of gas velocity and turbulence intensity in the ESP channels with six shaped corona electrodes, where $u_{\mathrm{e}}$ is the local velocity of the EHD flow.

It can be observed in Fig. 9 that in the ESP channels without the dust-laden gas flow, i.e., $u_{0}=0 \mathrm{~m} / \mathrm{s}$, a series of vortexes induced by the secondary flow exist between the discharge electrodes and the collecting plates. The vortex intensity in the channel with Knife-shaped electrode is the highest (Fig. 9(f)), then Round-shaped electrode (Fig. 9(a)), Star1 and the Trap are the moderate (Figs. 9(b) and 9(d)), and Star-2 is the weakest and it is similar to that in Square-shaped electrode (Figs. 9(c) and $9(\mathrm{e})$ ). Although the vortex intensity in Star-2 and the Square systems are only about half of that in the other four, the vortex regions of these two systems near the inlet and outlet are wider than those of the other discharge electrodes. In addition, there is a distinct low-speed vortex region near the collecting plate between two adjacent vortexes of Star-2 electrode, as shown in Fig. 9(c). The above analysis shows that the electrode shape affects the corona position on the electrode surface and the space 


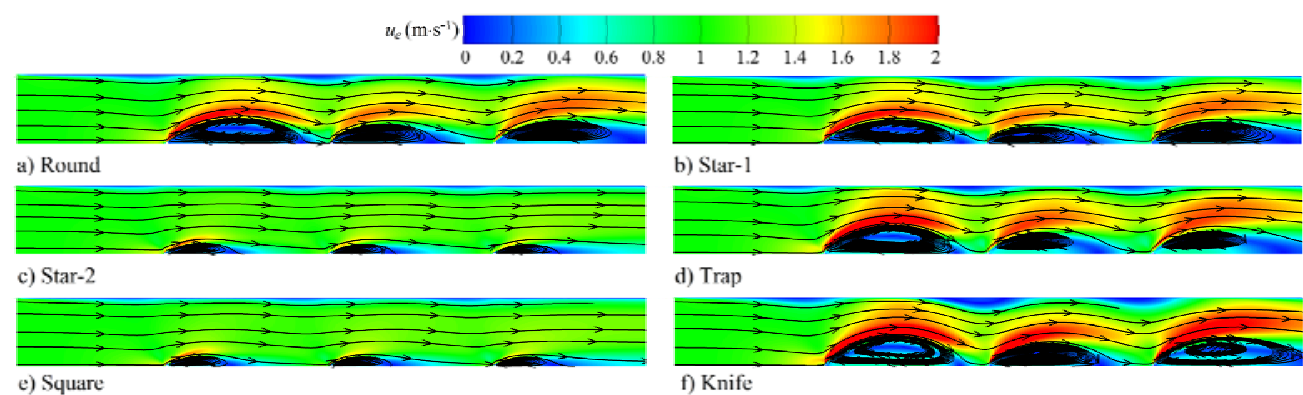

Fig. 12. Comparison of ionic wind induced flows between the six ESP channels at $u_{0}=1.0$ $\mathbf{m} / \mathbf{s}$.

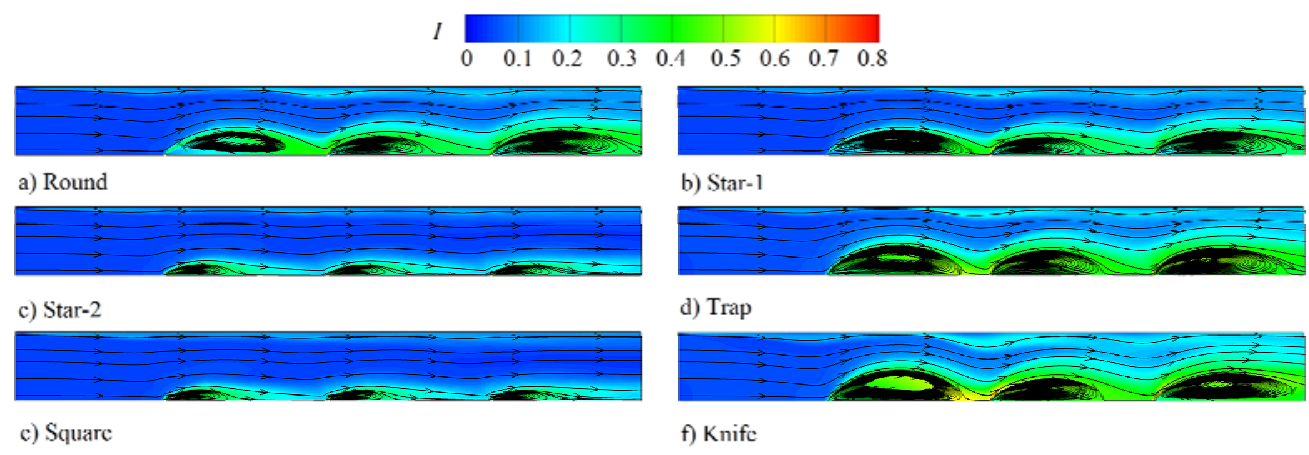

Fig. 13. Turbulence intensities in the six ESP channels at $u_{0}=1.0 \mathrm{~m} / \mathrm{s}$.

charge distribution, which in turn affects the intensity and the region of the vortex induced by the electric wind.

Vortexes electrohydrodynamically introduced by secondary flow exists not only at the downstream of the discharge electrodes, but also near the collecting plates when $u_{0}=0.5 \mathrm{~m} / \mathrm{s}$, which can be observed from Fig. 10. Turbulence intensity from Inlet to Outlet in the channel is increased markedly by secondary flow, shown in Fig. 11, and the turbulence intensity by the secondary flow at the downstream of the discharge electrodes is stronger than that near the collecting plate. The vortexes near the collecting plate at the upstream of Knife-shaped system offers the highest recirculation velocity, covers the widest area (see Fig. 10(f)) and produces the highest turbulence intensity (see Fig. 11(f)), followed by the Round, Star-1 and the Trap electrodes, and Star-2 discharge electrode is the weakest. For the vortex in the downstream of the discharge electrode, the strongest intensity, the widest region and the highest turbulence intensity are obtained by the Knife discharge electrode, and the weakest is that in the channel with the Square electrode though it is slightly weaker than that of Star-2 electrode.

When the inlet velocity is increased to $u_{0}=1.0 \mathrm{~m} / \mathrm{s}$, see in Figs. 12 and 13, the vortexes near the collecting plate in the all six channels disappear but still exist in the downstream of all the discharge electrodes, which increase the turbulence intensity in the core regions of the channels. Knife-shaped systems generate the highest vortex intensity and turbulence intensity behind the discharge electrodes, then Star-1, Trap and Round with the similar intensity, and the Star-2 and the Square electrodes are the two weakest cases and both are much less than the vortex intensity of Knife-shaped system.

It is well known that there are some important factors leading to the reentrainment of precipitated particles in the operating ESP channel (Parker 1997; Islamov 2018), one of which is the direct scouring action of the airflow on the plates. Comparisons of the area directly scoured by the airflow and the acceleration effect due to the electric wind at $u_{0}=$ $0.5 \mathrm{~m} / \mathrm{s}$ and $u_{0}=1.0 \mathrm{~m} / \mathrm{s}$ show that, as shown in Figs. 10 and 12, the Knife discharge electrode corresponds to the largest area scoured by the airflow and the most distinct acceleration of the main flow, next the Trap, and then the Round and Star-1 electrodes. The airflow acceleration in Knifeshaped system is much stronger than those in Star-2 and Square-shaped electrodes. The airflow velocity near the collecting surface of the Knife electrode is twice that of Star-2 and the Square discharge electrodes, meaning that the reentrainment of the Knife electrode by the airflow scouring directly would be the heaviest, then the Trap and the Round electrodes.

As the inlet velocity $u_{0}$ is increased to $2.0 \mathrm{~m} / \mathrm{s}$, Figs. 14 and 15 shows that the vortexes and the turbulence intensity become weak in the downstream of the discharge electrodes, and the 


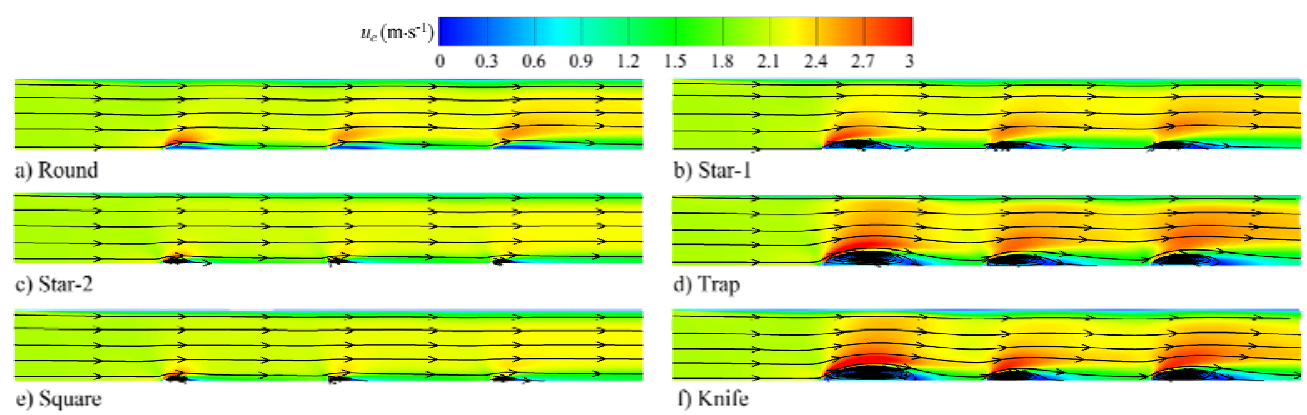

Fig. 14. Comparison of ionic wind induced flows between the six ESP channels at $u_{0}=2.0$ $\mathbf{m} / \mathbf{s}$.

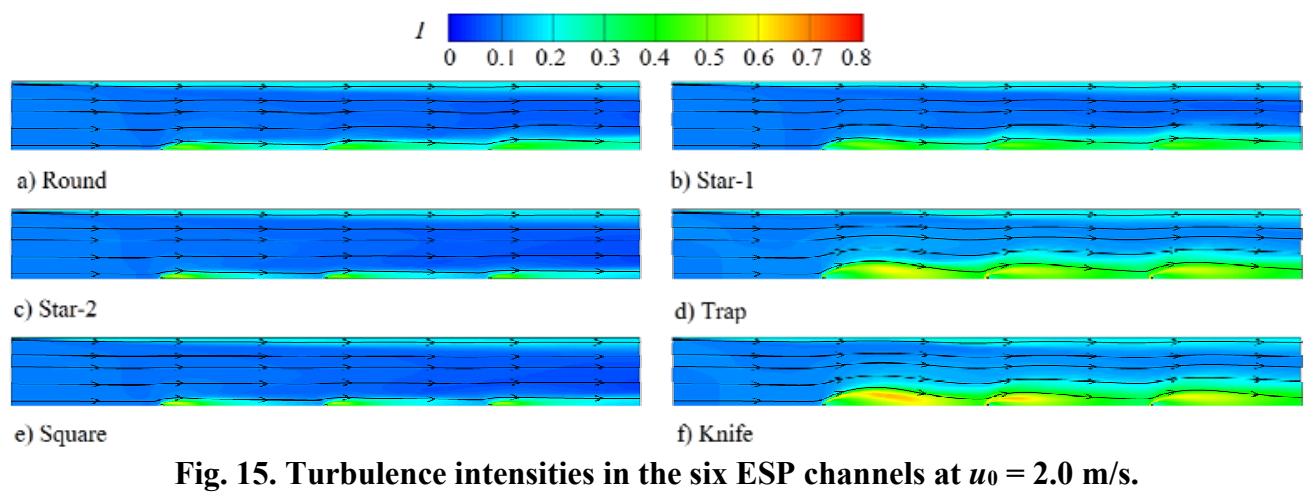

vortex intensity in the center of the channel with Knife-shaped electrode is the strongest, next are the Trap and Star-1 electrodes, and Star-2 and the Square electrodes are the weakest. The vortexes become narrow in the downstream of the discharge electrodes, i.e., the electric-wind effect is restricted to the near-wire region. Figure 14 also indicates that although the inlet velocity is increased to $2.0 \mathrm{~m} / \mathrm{s}$, the interaction between the primary flow and the electric wind still exists. For the corona wind velocity is less than $1.5 \mathrm{~m} / \mathrm{s}$ (Fig. 10), the secondary flow is now superimposed on the main flow. So the local velocity in most region of the channel is higher than the mean velocity after passing through the first corona electrode, which is consistent with the test data by Leonard et al. (1983).

In addition, it can be inferred from the results in Figs. 10, 12 and 14 that the vortexes in the vicinity of the collecting plate push the airflow away from the deposition planes to the center of the channel and this might decrease particle collection efficiency. On the contrary, the vortexes in the downstream of the discharge electrodes drive airflow toward the collecting surface and might increase the efficiency, which agrees fairly well with the results of Kallio and Stock (1992), Farnoosh et al. (2011) and Soldati (2000). These two phenomena exist simultaneously in the channel, but the impacts on the collection efficiency needs further study.

\section{Conclusion}

A numerical model with the consideration of space charge density distribution is developed to simulate the electrical characteristics and EHD flows in a plate-type ESP channel with six shapes of corona electrodes (i.e., the Round-, Star-1, Star-2, the Trap, the Square- and the Knife-shaped). The simulations indicate that the flow patterns in the channels are different for the six configurations, and, therefore, lead to the following conclusions:

(1) Corona positions of the discharge electrode determine the details of the space charge distribution, which form two different kinds of space charge distribution for the six shaped discharge electrodes. One is formed by two-point corona referred to the Trap, Star-2 and the Square discharge electrode, and the other is produced by the Knife, Star-1 and the Round with single-point corona.

(2) For the inlet velocity $u_{0}<1.0 \mathrm{~m} / \mathrm{s}$, the vortexes and the high turbulence induced remarkably by the electric wind exist not only in the downstream of the discharge electrodes, but also near the collecting plates. Moreover, the vortex near the collecting surface at the upstream of Knife-shaped system offers the highest strength, covers the widest region and produces the highest turbulence intensity, followed by the Round, Star-1 and the Trap 
electrodes, and the Square and Star-2 discharge electrode is the weakest.

(3) When the inlet velocity $u_{0}$ exceeds $1.0 \mathrm{~m} / \mathrm{s}$, the local recirculations and the high turbulence generated by the secondary flow near the collecting plate in the all six channels disappear but still remain in the downstream of the discharge electrodes, thus the airflow in the central region would be speeded up. The acceleration in Knifeshaped system is much higher than those in Star-2 and Square-shaped electrodes.

(4) As $u_{0}$ is increased to $2.0 \mathrm{~m} / \mathrm{s}$, the vortexes and the turbulence intensity produced by the secondary flow become weak and narrow in the downstream regions of the discharge electrodes. However, there is still a certain degree of interaction between the primary flow and the electric wind, and the local velocity in most region of the channel is higher than the mean velocity.

\section{ACKNOWLEDGEMENTS}

This work was supported by the National Natural Science Foundation of China (Grant No. 51578121) and the Chinese Universities Scientific Fund (Grant No. CUSF-DH-D-2017094).

\section{APPENDIX}

Supplementary UDF code regarding the interaction between the gas flow and the electric field was used for the simulations accompanies this paper, and is available for download from the Journal of Applied Fluid Mechanics webpage.

\section{REFERENCES}

Adamiak, K. (2013). Numerical models in simulating wire-plate electrostatic precipitators: a review. Journal of Electrostatics 71(4), 673-680.

Al-Hamouz, Z., A. El-Hamouz and N. Abuzaid (2011). Simulation and experimental studies of corona power loss in a dust loaded wire-duct electrostatic precipitator. Advanced Powder Technology 22(6), 706-714.

Brocilo, D., J. Podlinski and J. Mizeraczyk (2004). Electrohydrodynamic flow patterns in a wide spacing spike-plate electrostatic precipitators under negative corona. 5th International Conference on Applied Electrostatics, 100103.

Brocilo, D., J. S. Chang and R. D. Findlay (2001). Modeling of electrode geometry effects on dust collection efficiency of wire-plate electrostatic precipitators. Proceedings of 7 th International Conference on Electrostatic Precipitation, 1118.

Choi, B. S. and C. A. J. Fletcher (1998). Turbulent particle dispersion in an electrostatic precipitator. Applied Mathematical Modelling
22(12), 1009-1021.

Chun, Y. N., J. S. Chang and A. A. Berezin (2007). Numerical modeling of near corona wire electrohydrodynamic flow in a wire-plate electrostatic precipitator. IEEE Transactions on Dielectrics and Electrical Insulation 14(1), 119-124.

Farnoosh, N., K. Adamiak and G. S. P. Castle (2011). Three-dimensional analysis of electrohydrodynamic flow in a spiked electrode-plate electrostatic precipitator. Journal of Electrostatics 69(5), 419-428.

Feng, Z., Z. Long and K. Adamiak (2018). Numerical simulation of electrohydrodynamic flow and vortex analysis in electrostatic precipitators, IEEE Transactions on Dielectrics and Electrical Insulation 25(2), 404-412.

Fu, X., S. Wang and B. Zhao (2013). Emission inventory of primary pollutants and chemical speciation in 2010 for the Yangtze River Delta region, China. Atmospheric Environment 70, 39-50.

Islamov, R. S. (2018). Influence of gas velocity on the particle collection and reentrainment in an air-cleaning electrostatic precipitator. Aerosol Science and Technology 52(12), 1415-1428.

Jarzebski, S. and M. Chojnowski (1974). Influence of shapes of corona electrodes on density of emission currents in electrostatic dust collectors. Atmospheric Environment 8(1), 2940 .

Jędrusik, M., J. B. Gajewski and A. J. Świerczok (2001). Effect of the particle diameter and corona electrode geometry on the particle migration velocity in electrostatic precipitators. Journal of Electrostatics 51-52, 245-251.

Kallio, G. A. and D. E. Stock (1992). Interaction of electrostatic and fluid dynamic fields in wireplate electrostatic precipitators. Journal of Fluid Mechanics 240, 133-166.

Kim, Y. J., S. H. Jeong and W. S. Hong (1998). Effect of the plate spacing and discharge electrode shape on the efficiency of wide plate spacing electrostatic precipitator. Proceedings of 7th International Conference on Electrostatic Precipitation, 590-595.

Lagarias, J. S. (1960). Discharge electrodes and electrostatic precipitators. Journal of the Air Pollution Control Association 10(4), 271-274.

Lei, H., L. Z. Wang and Z. N. Wu (2008). EHD turbulent flow and Monte-Carlo simulation for particle charging and tracing in a wire-plate electrostatic precipitator. Journal of Electrostatics 66(3-4), 130-141.

Leonard, G. L., M. Mitchner and S. A. Self (1983). An experimental study of the electrohydrodynamic flow in electrostatic precipitators. Journal of Fluid Mechanics 127, 
H. Shen et al. / JAFM, Vol. 13, No. 6, pp. 1707-1718, 2020.

\section{$123-140$.}

Neimarlija, N., I. Demirdžić and S. Muzaferija (2011). Numerical method for calculation of two phase electrohydrodynamic flows in electrostatic precipitators. Numerical Heat Transfer, Part A: Applications 59(5), 321-348.

Park, S. J. and S. S. Kim (2000) Electrohydrodynamic flow and particle transport mechanism in electrostatic precipitators with cavity walls. Aerosol Science \& Technology 33(3), 205-221.

Parker, K. R., (1997). Applied Electrostatic Precipitation. Blackie Academic and Professional, New York, US.

Podlinski, J., A. Berendt and J. Mizeraczyk (2013). Electrohydrodynamic secondary flow and particle collection efficiency in spike-plate multi-electrode electrostatic precipitator. IEEE Transactions on Dielectrics and Electrical Insulation 20(5), 1481-1488.

Roache, P. J. (1994). Prespective: a method for uniform reporting of grid refinement studies. Journal of fluids engineering 116(3), 405-413.

Rouhollahi, R., S. B. Islami and R. Gharraei (2018) Application of electric field to developing falling films using wire-plate electrode configuration-An experimental study. Journal of Applied Fluid Mechanics 11(5), 1349-1363.

Sander, S., S. Gawor and U. Fritsching (2018). Separating polydisperse particles using electrostatic precipitators with wire and spiked-wire discharge electrode design. Particuology 38, 10-17.

Shen, H., W. Yu, H. Jia and Y. Kang (2018). Electrohydrodynamic flows in electrostatic precipitator of five shaped collecting electrodes. Journal of Electrostatics 95(10), 61-70.

Soldati, A. (2000). On the effects of electrohydrodynamic flows and turbulence on aerosol transport and collection in wire-plate electrostatic precipitators. Journal of Aerosol Science 31, 293-305.

Sugiyama, H., H. Ogura and T. Shiojima (2013). Simulation of electrohydrodynamic jet flow in dielectric fluids. Journal of Applied Fluid Mechanics 6(3), 405-412.

Sun, X., S. Cheng and J. Lang (2018). Development of emissions inventory and identification of sources for priority control in the middle reaches of Yangtze River Urban Agglomerations. Science of The Total Environment 625, 155-167.

Tathiri, G., H. Parishani and E. Esmaeilzadeh (2017). A finite volume study on the effects of electro-hydro-dynamic fluid acceleration on airflow around a cylinder at low Reynolds numbers. Journal of Applied Fluid Mechanics 10(3), 889-897.

White, H. J. (1963). Industrial electrostatic precipitation. Addison-Wesley Pub. Co.. London, US.

Xue, Y., H. Tian and J. Yan (2016). Temporal trends and spatial variation characteristics of primary air pollutants emissions from coalfired industrial boilers in Beijing, China. Environmental Pollution 213, 717-726.

Yamamoto, T. and H. R. Velkoff (1981). Electrohydrodynamics in an electrostatic precipitator. Journal of Fluid Mechanics 108, $1-18$ 